But some experts say it may be premature to say that investments at the local level necessarily yield improvements in patient care.

Robin Tamblyn, scientific director of the Clinical and Health Informatics Research Group at McGill University in Montréal, Quebec, says the Ontario survey warrants follow-up research.

Because it is based on survey responses from a subset of Ontario users, the study might not be usable "for an argument on return on investment," she cautions.

But the finding that physicians widely endorse EMR usage should prompt closer investigation of how the use of EMRs affects such things as medical testing procedures, pharmaceuticals and specialist referrals, she adds.

Similarly, Anne Holbrook, principal investigator on a series of electronic health research studies at McMaster University in Hamilton, Ontario, cautions that the OntarioMD survey data is drawn from "only a portion of all docs using EMRs" and contains "a major response bias" in that respondents had all received funds from the survey manager. As well, she warns that OntarioMD is in a potential conflict of interest "as their survival depends on this evolution of EMRs."

Nonetheless, the results of the survey "make a lot of sense," she says, adding that EMRs appear to be per- forming the basic functions they were intended to perform.

Holbrook also notes that surveying physician attitudes, alone, is not an indicator of improvements in patient care. "It is much more important to actually measure whether quality of care is improved and whether patient safety is improved. This requires prospective, unbiased studies looking at clinical patient outcomes."

Canada Health Infoway declined interview requests on whether its strategy should be revised to focus more squarely on programs that promote use of EMRs at the physician level. - Paul Christopher Webster, Toronto, Ont.

CMAJ 2011.DOI:10.1503/cmaj.109-3744

\title{
End-of-life planning framework calls for fewer checklists
}

A national framework for advance care planning would see Canada's "tick-box" approach to preparing for the worst incorporated into a more formal process of ongoing communication and reflection between patients, their families and health care providers.

The framework, now in development by the Canadian Hospice Palliative Care Association (CHPCA), aims to shift the focus of advance care planning from patients making lists of the medical interventions they'd like to receive or refuse to a more flexible conversation about their values and the goals of care.

"Two years ago, people thought simply filling out a form was adequate advance care planning. Get the patient's wishes down on paper and you're good to go," says Louise Hanvey, project leader of the team developing the framework.

It's never been easier for Canadians to give instructions about the kind of care they'd want to receive but the extent to which those are followed, or are even useful, is limited, Hanvey says, largely because of a lack of awareness, training and infrastructure to support the communication required for effective advance care planning.

"We developed the forms to create these documents and the provinces got the legal supports in place to recognize them, but we didn't develop the public education, professional engagement or system supports to use them effectively," Hanvey explains.

According to a draft of the framework, effective advance care planning requires a number of conversations over

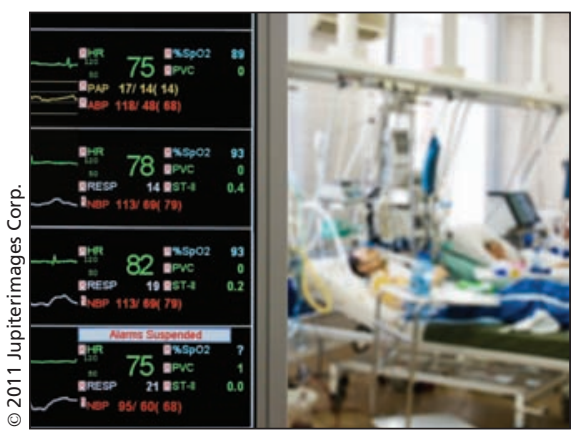

End-of-life care often isn't broached until too late, when a crisis occurs or life-sustaining treatments have already been instituted despite a poor prognosis.

time so that patients can articulate and clarify their wishes. Those should occur in a nonstressful environment and patients should craft written directions in consultation with their health care team and legal advisors. By these standards, most advance care planning in Canada is poorly executed, says Hanvey. End-oflife care typically isn't broached until too late and many directives are not adequately prepared, communicated or utilized (www.chpca.net/projects/advance _care_planning/acp_environmental_scan _sept_9_09.pdf).

The draft framework highlights the role that family doctors can play in encouraging advance care planning conversations before the diagnosis of a chronic or terminal illness. But Hanvey says health care professionals often lack the training to actively initiate those conversations.

The draft framework also recommends integrating advance care planning core competencies into undergraduate and graduate education; training frontline staff to routinely check if patients have plans or directives; and developing online continuing education programs for health care providers. It also calls on the provincial-territorial ministries of health to provide resources to compensate physicians for time spent on advance care planning.

The draft framework notes that effective advance care planning has been linked to increased patient and family satisfaction with care, fewer hospitalizations and less resource use in nursing homes, fewer life-sustaining procedures, lower rates of intensive care unit admissions and, for advanced cancer patients, substantially lower health care costs in the final week of life. The final version of the framework will be released "in the near future." - Lauren Vogel, CMAJ

CMAJ 2011.DOI:10.1503/cmaj.109-3746 